\title{
EDITORIAL Where did the ventricles go?
}

\section{HIGHLIGHT}

\author{
Richard F. Keep, ${ }^{*} \dagger$ Jianming Xiang* and Anuska V. Andjelkovic* + \\ *Department of Neurosurgery, University of Michigan, Ann Arbor, Michigan, USA \\ $\dagger$ Department of Molecular \& Integrative Physiology, University of Michigan, Ann Arbor, Michigan, \\ USA
}

$\ddagger$ Department of Pathology, University of Michigan, Ann Arbor, Michigan, USA

Read the full article 'Molecular heterogeneity in the choroid plexus epithelium: the 22-member $\gamma$-protocadherin family is differentially expressed, apically localized, and implicated in CSF regulation' on page 913.

The protocadherin (Pcdh) family of genes is a large subgroup within the cadherin superfamily of cell adhesion molecules. The Pcdh family is divided into clustered (Pdch- $\alpha$, Pdch- $\beta$ and Pdch- $\gamma$ genes) and non-clustered families and they are predominantly expressed in brain (Yagi 2008). There has been considerable interest in the role of the Pdch family in synaptogenesis and neuronal survival (Weiner et al. 2005). However, in a paper in this issue of Journal of Neurochemistry, Lobas et al. (2012) have surprisingly found that all 22 of the $\gamma$-Pdchs are expressed at the choroid plexus, the site of the blood-CSF barrier. In addition, they found that these $\gamma$-Pdchs were present at the apical (CSF-facing) membrane of the choroid plexus epithelium, where there is very limited expectation of cell-cell contact, and that there was considerable variation in expression between individual epithelial cells. They were also surprised to find that targeted mutation of the Pcdh- $\gamma$ locus resulted in a marked reduction in the volumes of the cerebral ventricles, suggesting that $\gamma$-Pdchs have a major impact on CSF production. Devising methods to modulate CSF formation is clinically very important, particularly with respect to hydrocephalus, and understanding the effects of $\gamma$-Pdchs may help in developing new therapeutics. Currently, the principal treatment for hydrocephalus is placement of a shunt and $40-50 \%$ of shunts fail within 2 years of surgery (Notarianni et al. 2009).

It is noticeable that the reduction in ventricle volume found with targeted mutation of the Pcdh- $\gamma$ locus is very similar to that found in the $\mathrm{NBCn} 2 \mathrm{KO}$ mouse that is thought to be involved in CSF secretion (Jacobs et al. 2008). CSF secretion involves the transport of primarily $\mathrm{Na}^{+}, \mathrm{Cl}^{-}$and $\mathrm{HCO}_{3}{ }^{-}$, and water, from blood across the choroid plexus epithelium to the cerebral ventricles (Brown et al. 2004). That vectoral transport involves a polarized distribution of ion transporters and channels at the choroid plexus epithelium. For example, $\mathrm{Na}^{+}-\mathrm{K}^{+}-\mathrm{ATPase}$, the $\mathrm{Na}^{+}-\mathrm{K}^{+}-2 \mathrm{Cl}^{-}$ cotransporter, $\mathrm{NKCC} 1$, and the $\mathrm{Na}^{+} / \mathrm{H}^{+}$exchanger, NHE1, have an apical distribution (CSF facing), whereas the anion exchanger, $\mathrm{AE} 2$, the $\mathrm{Na}^{+} / \mathrm{HCO}_{3}{ }^{-}$-cotransporter, $\mathrm{NBCn} 1$, and the $\mathrm{Na}^{+} / \mathrm{Cl}^{-} / \mathrm{HCO}_{3}{ }^{-}$transporter, $\mathrm{NBCn} 2$, are present on the basolateral membrane (blood facing) of the epithelium (Damkier et al. 2010). Targeting specific transporters can reduce CSF production (e.g. inhibiting $\mathrm{Na}^{+}-\mathrm{K}^{+}$-ATPase causes marked reductions; Brown et al. 2004). Furosemide with acetazolamide (which indirectly affects transport) has been used clinically to reduce CSF production in hydrocephalus. However, these drugs have effects other than on the choroid plexus (e.g. metabolic and cerebrovascular effects, and nephrocalcinosis) and their use has been questioned (Shooman et al. 2009). Developing methods of specifically targeting choroid plexus ion transport would have great clinical importance. If specific $\gamma$-Pcdh are involved in regulating CSF secretion, as Lobas et al. (2012) suggest, this might provide an alternate strategy to transport inhibitors.

Of course, the big question is, how does the $\gamma$-Pcdh KO result in such changes in ventricle size? There were no obvious changes in choroid plexus morphology, but there are a number of $\gamma$-Pcdh interactions that might affect choroid plexus function (Fig. 1). $\gamma$-Protocadherins can undergo homotypic trans-interactions with other cells. However, given the apical (CSF-facing) distribution of $\gamma$-Pcdhs at the choroid plexus, this seems unlikely, although there are sporadic immune cells on the apical surface. $\gamma$-Protocadherins

Received November 14, 2011; accepted November 14, 2011.

Address correspondence and reprint requests to Richard F. Keep, PhD, R5018 Biomedical Science Research Building, University of Michigan, 109 Zina Pitcher Place, Ann Arbor, Michigan 48109-2200, USA.

E-mail: rkeep@umich.edu

Abbreviations used: Pcdh, protocadherin. 


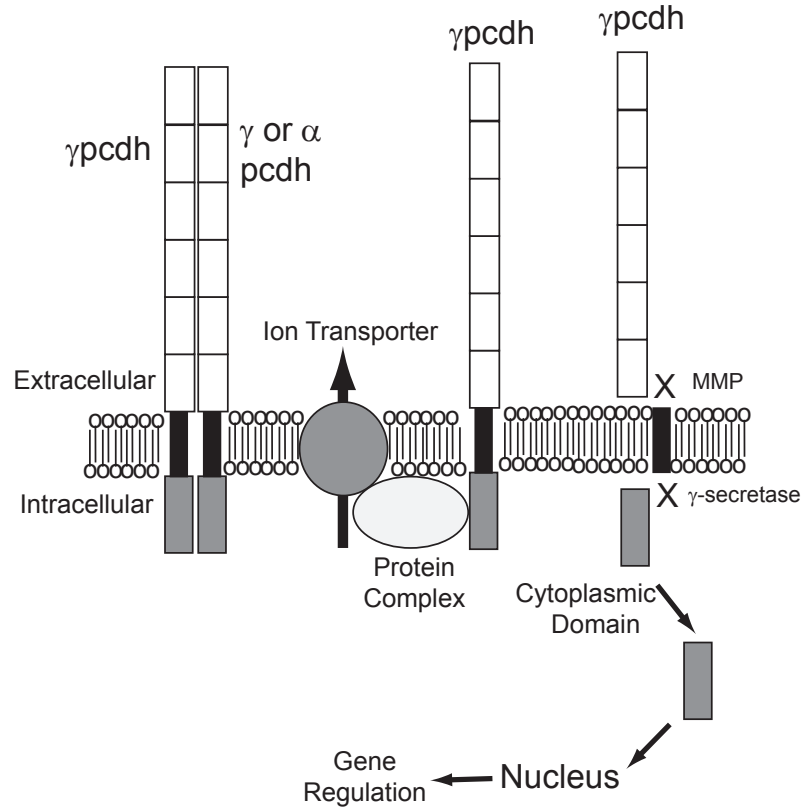

Fig. 1 A schematic of $\gamma$-pcdh interactions. They undergo homophilic trans- and cis-interactions as well as heterophilic cis-interactions with other $\gamma$-Pcdhs (Schreiner and Weiner 2010) (only cis-interactions are shown on the figure). They can also undergo cis-interactions with $\alpha$-Pcdhs, which in turn may interact with integrins (Yagi 2008). $\gamma$-Pcdhs can also interact with a wide array of other types of proteins, including regulators of ion transport, forming a multiprotein complex (Han et al. 2010). In addition, $\gamma$-Pcdhs undergo cleavage (X) of the extracellular domain by metalloproteinase (MMP) and the cytoplasmic domain by $\gamma$-secretase (Yagi 2008). The cytoplasmic domain can travel to the nucleus where it may regulate transcription (Yagi 2008). Multi-protein complexes and transcriptional regulation could be ways whereby choroid plexus transport is regulated by $\gamma$-Pcdhs.

are also capable of $c i$-interactions with other $\gamma$ - and $\alpha$-Pcdhs (Yagi 2008; Schreiner and Weiner 2010) and those complexes might affect choroid plexus function. However, it should also be noted that the cytoplasmic terminal of Pcdhs can interact with multiple proteins and this might impact CSF secretion. Potential interactions are emphasized by a recent proteomic analysis of $\gamma$-Pcdh-associated protein complexes which identified 154 non-redundant proteins (Han et al. 2010). These included proteins involved in cytoskeleton, signaling, trafficking/transport, nucleic acid/protein processing and metabolism. Interestingly, they included members of the FXYD family of ion transport regulators. One member of that family, phospholemman, is known to be highly expressed at the choroid plexus (Feschenko et al. 2003) where it is thought to regulate $\mathrm{Na}^{+}-\mathrm{K}^{+}$-ATPase activity. In Usher's syndrome, the leading cause combined deaf-blindness in humans, mutations in one of a series of proteins involved in a protein network can cause senso-neuronal degeneration. Two of the proteins in that network are pcdh 15 and the sodium-bicarbonate transporter, NBC3 (Reiners et al. 2006), again suggesting that pcdhs may have a role in regulating ion transport. It should also be noted that the $\gamma$-Pcdh cytoplasmic domain is subject to cleavage by $\gamma$-secretase and the derived fragment is involved in gene regulation (Yagi 2008).

Understanding how the $\gamma$-Pcdhs affect ventricular volume requires much further work but that should produce greater insight into the function of $\gamma$-Pcdhs and their mechanisms of action. In addition, building on the very novel findings of Lobas et al. (2012) should provide insight into how CSF production is and may be regulated.

\section{References}

Brown P. D., Davies S. L., Speake T. and Millar I. D. (2004) Molecular mechanisms of cerebrospinal fluid production. Neuroscience 129, 957-970.

Damkier H. H., Brown P. D. and Praetorius J. (2010) Epithelial pathways in choroid plexus electrolyte transport. Physiology 25, 239249.

Feschenko M. S., Donnet C., Wetzel R. K., Asinovski N. K., Jones L. R. and Sweadner K. J. (2003) Phospholemman, a single-span membrane protein, is an accessory protein of Na,K-ATPase in cerebellum and choroid plexus. J. Neurosci. 23, 2161-2169.

Han M.-H., Lin C., Meng S. and Wang X. (2010) Proteomics analysis reveals overlapping functions of clustered protocadherins. Mol. Cell Proteomics 9, 71-83.

Jacobs S., Ruusuvuori E., Sipila S. T. et al. (2008) Mice with targeted Slc4a10 gene disruption have small brain ventricles and show reduced neuronal excitability. Proc. Natl Acad. Sci. USA 105, 311316.

Lobas M. A., Helsper L., Vernon C. G., Schreiner D., Zhang Y., Holtzman M. J., Thedens D. R. and Weiner J. A. (2012) Molecular heterogeneity in the choroid plexus epithelium:the 22-member $\gamma$-protocadherin family is differentially expressed, apically localized, and implicated in CSF regulation. J. Neurochem. 120, 913-927.

Notarianni C., Vannemreddy P., Caldito G., Bollam P., Wylen E., Willis B. and Nanda A. (2009) Congenital hydrocephalus and ventriculoperitoneal shunts: influence of etiology and programmable shunts on revisions. J. Neurosurg-Pediatr. 4, 547-552.

Reiners J., Nagel-Wolfrum K., Jurgens K., Marker T. and Wolfrum U. (2006) Molecular basis of human Usher syndrome: Deciphering the meshes of the Usher protein network provides insights into the pathomechanisms of the Usher disease. Exp. Eye Res. 83, 97-119.

Schreiner D. and Weiner J.A. (2010) Combinatorial homophilic interaction between gamma-protocadherin multimers greatly expands the molecular diversity of cell adhesion. Proc. Natl Acad. Sci. USA 107, 14893-14898.

Shooman D., Portess H. and Sparrow O. (2009) A review of the current treatment methods for posthaemorrhagic hydrocephalus of infants. CSF Res. 6, 1.

Weiner J. A., Wang X., Tapia J. C. and Sanes J. R. (2005) Gamma protocadherins are required for synaptic development in the spinal cord. Proc. Natl Acad. Sci. USA 102, 8-14.

Yagi T. (2008) Clustered protocadherin family. Dev. Growth Differ. 50(Suppl. 1), S131-S140. 